Research Article

\title{
Survival Status and Predictors of Mortality among Multidrug-Resistant Tuberculosis Patients in Saint Peter's Specialized Hospital, Addis Ababa, Ethiopia
}

\author{
Mikyas Arega Muluneh $\mathbb{D D}^{1}$, Abayneh Birlie Zeru $\mathbb{D}^{2},{ }^{2}$ Behailu Tariku Derseh ${ }^{(\mathbb{D}},{ }^{2}$ \\ and Abebaw Molla Kebede ${ }^{3}{ }^{3}$ \\ ${ }^{1}$ Department of Midwifery, Institute of Medicine and Health Sciences, Debre Berhan University, Debre Berhan, Ethiopia \\ ${ }^{2}$ Department of Public Health, Institute of Medicine and Health Sciences, Debre Berhan University, Debre Berhan, Ethiopia \\ ${ }^{3}$ Department of Nutrition and Reproductive Health, School of Public Health, Mizan-Tepi University, Tepi, Ethiopia
}

Correspondence should be addressed to Mikyas Arega Muluneh; miky24real@gmail.com

Received 16 December 2020; Accepted 21 August 2021; Published 6 September 2021

Academic Editor: Mohammad Sistanizad

Copyright $\odot 2021$ Mikyas Arega Muluneh et al. This is an open access article distributed under the Creative Commons Attribution License, which permits unrestricted use, distribution, and reproduction in any medium, provided the original work is properly cited.

\begin{abstract}
Background. Multidrug-Resistant Tuberculosis (MDR-TB) is tuberculosis that is resistant to at least both rifampicin and isoniazid. The World Health Organization as reported in 2019 revealed that Ethiopia is among the 20 countries with the highest estimated numbers of incident MDR-TB cases. However, supporting evidence is limited in the study area after the Ethiopian national strategic plan for tuberculosis prevention and control is started. Objective. To determine survival status and predictors of mortality among multidrug-resistant tuberculosis patients treated in Saint Peter's Specialized Hospital at Addis Ababa, Ethiopia, 2020. Methods. An institutional retrospective cohort study was conducted using all MDR-TB patients who were enrolled in Saint. Peter's Specialized Hospital from January 01, 2015, to December 31, 2017. A pretested data extraction form that had 5 items for sociodemographic and 15 items for the measurement of clinical characteristics of 484 MDR-TB patients was used. STATA software version 14.2 was used for data cleaning and analysis. A variable that fitted in the bivariable Cox proportional hazard model at $p$ value $<0.25$ was used in the final multivariable Cox proportional hazard model, and independent predictors of time to event were determined at a $p$ value of 0.05 . Result. A total of 484 patients were followed up for 5,078 person-months. Among the total patients, nearly half, 238 (48.8\%), were males. The median age of patients was 30 years (interquartile range (IQR), 24-39), and $56(11.6 \%)$ were aged between 1 and 19 years. During the follow-up period, 315 (65.1\%) patients were cured, 125 (25.8\%) completed treatment, $24(5 \%)$ died, and $20(4.1 \%)$ were lost to follow-up. The overall cumulative probability survival of the patients at the end of treatment was $94.85 \%$ (95\% confidence interval (CI): $92.38 \%-96.53 \%$ ). The independent predictors of time to death were being anemic $(\mathrm{AHR}=3.65 ; 95 \% \mathrm{CI}: 1.36,9.79)$, having clinical complication $(\mathrm{AHR}=3 ; 95 \% \mathrm{CI}: 1.2$, 7.5), and being HIV infected $(\mathrm{AHR}=5.8 ; 95 \% \mathrm{CI}: 2.2,15.7)$. Conclusions. MDR-TB patients' survival rate was high in St Peter's Specialized Hospital. MDR-TB patients with anemia, HIV coinfection, and clinical complications had higher risk of mortality. So, prevention and controlling of anemia, HIV/AIDS, and clinical complications will reduce the mortality of MDR-TB patients.
\end{abstract}

\section{Introduction}

Multidrug-resistant tuberculosis (MDR-TB) is tuberculosis (TB) disease resistant to at least both rifampicin and isoniazid, the two most powerful anti-TB drugs. Detection of MDR-TB requires bacteriological confirmation of TB and testing for drug resistance using rapid molecular tests, culture methods, or sequencing technologies. Treatment requires a course of second-line drugs at least for 9 months and up to 20 months, supported by counseling and monitoring for adverse outcomes $[1,2]$.

$\mathrm{TB}$ remains a major cause of ill health and is one of the top 10 causes of death worldwide. An estimated 10.0 million people fell ill with TB in 2018. Globally, there were 1.2 
million TB deaths among HIV-negative people in 2018 and an additional 251000 deaths among HIV-positive people. Since 2007, TB has been the leading cause of death from a single infectious agent, ranking above HIV/AIDS. In 2018, there were an estimated 390,000 new cases of multidrugresistant TB [1].

An anti-TB drug resistance surveillance data estimated $4.1 \%$ of new and $19 \%$ of previously treated TB cases in the world to have either multidrug-resistant or rifampicin-resistant tuberculosis (MDR/RR-TB). In 2016, an estimated 600,000 new cases of MDR/RR-TB emerged globally and caused 240,000 deaths. About $6.2 \%$ of MDR-TB cases have additional drug-resistance, extensively drug-resistant TB (XDR-TB) [3].

World Health Organization reports in 2019 reveal that Ethiopia is among the 20 countries with the highest estimated numbers of incident MDR-TB cases and listed among 14 countries that are the highest for $\mathrm{TB}, \mathrm{TB} / \mathrm{HIV}$, and MDR$\mathrm{TB}$ cases [1]. A major barrier to progressing toward TB elimination in Ethiopia is the TB "case detection rate" of only 60 percent, meaning that an estimated 80,000 Ethiopians who developed TB in 2014 were never diagnosed or treated, leading to ongoing spread of TB to family members and communities. The gap in case detection rate is even worse for the more severe MDR-TB, where less than a quarter of an estimated 2,200 Ethiopian MDR-TB patients are identified each year [4].

Different factors have been associated with mortality of MDR-TB patients. Some studies show that clinical complication, the presence of any chronic disease including HIV [5-7], older age [7,8], therapeutic delay $[5,7]$, smoking [5], body mass index (BMI) less than 18 [9], poor drug adherence [6], alcohol use [7], hypokalemia [8], low CD4 [9], extra-pulmonary TB [6], and living in rural areas [8] are risk factors for the mortality of MDR-TB patients. However, contradictory evidence revealed that BMI, HIV status, extra-pulmonary TB [8], and smoking [7] are not risk factors for mortality of MDR-TB patients. So, the purpose of this study was to assess survival status and identify predictors of mortality among MDR-TB patients in Saint Peter's Specialized Hospital, Addis Ababa, Ethiopia.

\section{Methods and Materials}

2.1. Study Design and Period. An institution-based retrospective cohort study design was used to assess the survival status and predictors of mortality among MDR-TB patients treated in Saint Peter's Specialized Hospital, Addis Ababa, Ethiopia, in March, 2020.

2.2. Study Setting and Population. The study was conducted in Addis Ababa, the capital city of Ethiopia. It is the largest city in Ethiopia, established in 1887 by emperor Menilik II. It has the status of both a city and a state. The city is divided into ten subcities. In 2017, it had a projected 3,435,028 million population of whom $1,809,577$ were females and the rest $1,625,451$ were males [10].
The study was conducted in Saint Peter's Specialized Hospital, the largest TB referral center in the country, in Addis Ababa. The hospital has a long history of TB management since it is a hospital where TB case management was initiated for the first time in the country in June 1961. The study population was MDR-TB patients who started MDR-TB treatment from January 1, 2015, to December 31, 2017 , and then, by considering the lately registered patients, we followed them up to the end of their treatment follow-up based on records which take up to December 2019. Five hundred and nineteen MDR-TB patients were managed at inpatient and outpatient levels during the indexed period.

All completely registered documents that contain identified variables of treated patients at Saint Peter's Specialized Hospital during the index period were included in the study. All transfer-out patients' documents whose treatment outcome was not available were excluded. By excluding 28 transfer outpatients and 7 patients with incomplete medical records, all the remaining $484 \mathrm{MDR}-\mathrm{TB}$ patients managed form 2015-2017 were included in the study.

2.3. Data Collection Tool and Procedures. A structured data extraction form was prepared in English language by reviewing previous similar works of literature and considering the availability of variables on medical registration books and treatment cards $[5,8,11-13]$. There were 5 items for sociodemographic and 15 items for the measurement of clinical characteristics of MDR-TB patients. The baseline weight of patients was taken as recorded on the patient card nearest to 0.5 kilograms. Baseline weight was categorized using the median weight of patients. Nothing was written about how the weight was measured. The hemoglobin level was recorded to the nearest $0.1 \mathrm{~g} / \mathrm{dl}$. The CD4 count of HIV/ AIDS patients was measured in number of cells per cubic millimeter. The person-time of patients was measured from the time of diagnosis of MDR-TB to event (death). A pretest was conducted on records of $25 \mathrm{MDR}-\mathrm{TB}$ patients that started treatment in 2014 at St. Peter's Specialized Hospital. Then, drug adherence and height variables were excluded because more than half of the records missed these variables.

Data were extracted by one BSc nurse who has been working at St. Peter's Specialized Hospital and 2 general practitioners (GPs), new graduates from Debre Berhan University.

\subsection{Operational Definition}

(i) Death (event): a patient who died of any cause during the course of MDR-TB treatment [14]

(ii) Censored: when the outcome of interest has not been observed for an individual; this includes cured completed, treatment failure, and defaulter [15]

(iii) Cured: treatment completed according to national recommendation without evidence of failure and three or more consecutive cultures taken at least 30 
days apart are negative after the intensive phase [14]

(iv) Treatment completed: treatment completed according to national recommendation without evidence of failure but no record that three or more consecutive cultures taken at least 30 days apart are negative after the intensive phase [14]

(v) Treatment success: sum of cured and completed treatment [14]

(vi) Treatment failure: treatment terminated or need for permanent regimen change of at least two antiTB drugs at the end of the intensive phase or later during treatment [14]

(vii) Defaulter (lost follow-up): a patient whose treatment was interrupted for two consecutive months or more for any reason without medical approval $[14,16]$

(viii) Time to death: time to occurrence of death measured from confirmed diagnosis of MDR-TB to event (death) (40)

(ix) Therapeutic delay: a confirmed MDR-TB patient who starts treatment after one month [5]

(x) Previously NOT treated for TB: patients have never been treated for $\mathrm{TB}$ or have taken anti-TB drugs for less than a month [14]

(xi) Previously treated case: a patient who admits having been treated for TB for one month or more [14]

(xii) Clinical complications: disorders that occur during the course of MDR-TB treatment

(xiii) Anemia: $<13 \mathrm{~g} / \mathrm{dl}$ for adult men, $<11 \mathrm{~g} / \mathrm{dl}$ for age 6 months to 5 years, and $<12 \mathrm{~g} / \mathrm{dl}$ for nonpregnant women and children aged 6-14 years were applied [17]

2.5. Data Quality Control. Orientation was given for half a day on the objectives and relevance of the study, confidentiality of information, and how to extract data on MDRTB logbook and treatment cards. Daily supervision of the data collection process and checking the completeness and consistency collected data were carried out by the principal investigator.

2.6. Data Processing and Analysis. First, data were checked for completeness and consistency before entering into computer. Then, data were coded, entered into Epi-Data version 3.1 Software, and exported to STATA statistical software version 14.2 for data cleaning and analysis.

A Shapiro-Wilk test $(p$ value $<0.05)$ showed that age, baseline weight, hemoglobin, and CD4 counts were not normally distributed. The descriptive analysis such as frequency distribution, percentages, and measures of central tendency for continuous variables (median and interquartile range (IQR)) was used. The maximum variance inflation factor was 1.26 among variables in the final model. A
Kaplan-Meier Survival curves were carried out to compare survival probability between groups using a log-rank test. The incidence of death with respect to person-time at risk was calculated. A variable that fitted in the bivariable Cox proportional hazard model at $p$ value $<0.25$ was used in the final multivariable Cox proportional hazard model, and independent predictors of time to event were determined at a $p$ value of $<0.05$.

The value of Harrell's C was 0.9638 for the assessment of model adequacy. The proportionality assumptions to the Cox proportional hazard model were checked using the goodness-of-fit (GOF) test by Schoenfeld residual. All candidate covariates for multivariable Cox regression did not violate the proportionality assumptions.

The goodness of fit of the final model was checked by likelihood ratio. Results of the likelihood ratio test were a chi-square of 104.47 with a $p$ value of $<0.0001$ suggesting that the model was good fitted.

Crude and adjusted hazard ratios with 95\% CI were calculated to measure the magnitude of the association between the covariates and time to MDR-TB death. A $p$ value of $<0.05$ was taken as a cutoff point to identify statistically significant variables.

\section{Result}

3.1. Sociodemographic Profile. The median age of patients was 30 years (IQR, 24-39), and 56 (11.6\%) were aged between 1 and 19 years. Among the total patients, over half of the patients, 248 (51.2\%), were females. A majority, 401 $(82.9 \%)$, were urban dwellers. More than one-third of patients, 185 (38.2\%), were government employees followed by $98(20.2 \%)$ students.

About three-fifths of the patients $(297,61.4 \%)$ and a quarter $(120,24.8 \%)$ were from Addis Ababa and Oromia region, respectively (Table 1 ).

3.2. Clinical Characteristics and Comorbidities. Near to three-fourth, 359 (74.5\%), of the patients were diagnosed through Gene Xpert followed by $72(14.9 \%)$ culture, 46 (9.5\%) line probe assay, and 7 (1.4\%) clinical method. More than three-fourth, 389 (80.4\%), of the MDR-TB patients had pulmonary TB. Almost one-third, 155 (32.0\%), of the MDRTB patients were new cases without previous history of antiTB medication. At the commencement of treatment, the median baseline weight was $45.5 \mathrm{~kg}$ (IQR: $40-52 \mathrm{~kg}$ ) and 172 (35.5\%) had less than $43 \mathrm{~kg}$. Regarding the history of substance use, three-fourths, 363 (75.0\%), were nonusers, 105 $(21.7 \%)$ were alcohol users, and 14 (2.9\%) were khat chewers.

A majority $(392,81.0 \%)$ of the patients started treatment on the same day of a confirmed diagnosis, 91 (18.8\%) within 10 days, and $1(0.2 \%)$ after 30 days of confirmed MDR-TB diagnosis. Almost all MDR-TB patients 463 (95.7) developed adverse drug reactions during the course of treatment. Of 484 MDR-TB patients, 96 (19.8\%) had at least one clinical complication throughout the course of treatment. The leading complication was pneumonia 59 (12.2\%) followed by hemoptysis 16 (3.3\%). 
TABLE 1: Sociodemographic characteristics of MDR-TB patients in SPSH, $2020(n=484)$.

\begin{tabular}{|c|c|c|c|}
\hline Variables & & Frequency & Percent \\
\hline \multirow{5}{*}{$\begin{array}{l}\text { Age at diagnosis } \\
\text { (years) }\end{array}$} & $\leq 24$ & 125 & 25.8 \\
\hline & $25-30$ & 131 & 27.1 \\
\hline & $31-40$ & 127 & 26.2 \\
\hline & $\geq 41$ & 101 & 20.9 \\
\hline & Median age (IQR) & $30(24-39)$ & - \\
\hline \multirow{2}{*}{ Sex } & Male & 236 & 48.8 \\
\hline & Female & 248 & 51.2 \\
\hline \multirow{7}{*}{ Region } & Addis Ababa & 297 & 61.4 \\
\hline & Oromia & 120 & 24.8 \\
\hline & Amhara & 38 & 7.9 \\
\hline & Tigray & 9 & 1.9 \\
\hline & Somali & 9 & 1.9 \\
\hline & SNNPR & 9 & 1.9 \\
\hline & Afar & 2 & 0.4 \\
\hline \multirow{2}{*}{ Residency } & Urban & 401 & 82.9 \\
\hline & Rural & 83 & 17.1 \\
\hline \multirow{7}{*}{ Occupation } & $\begin{array}{l}\text { Government } \\
\text { employee }\end{array}$ & 185 & 38.2 \\
\hline & Student & 98 & 20.2 \\
\hline & Housewife & 59 & 12.2 \\
\hline & Farmer & 57 & 11.8 \\
\hline & Merchant & 53 & 11.0 \\
\hline & Self-employed & 20 & 4.1 \\
\hline & Others & 32 & 2.5 \\
\hline
\end{tabular}

As shown in Table 2, all MDR-TB patients had been tested for HIV and $96(19.8 \%)$ were HIV positive. The median CD4 count of HIV-infected patients was 165 cells/ $\mathrm{mm}^{3}$ with a range of $46-800$ cells $/ \mathrm{mm}^{3}$. All HIV coinfected patients were on first-line ART drug regimen; 92 (96.8\%) were taking tenofovir disoproxil fumarate-lamivudine-efavirenz (TDF-3TC-EFV), and 3 (3.2\%) were on azidothymidine-lamivudine-efavirenz (AZT-3TC-EFV) drug regimen. One HIV-positive MDR-TB patient was not started $\mathrm{ART}$ at the time of diagnosis.

The median baseline hemoglobin level was $13.4 \mathrm{~g} / \mathrm{dl}$ (IQR: $12.2-14.5 \mathrm{~g} / \mathrm{dL}$ ), and more than a quarter of patients $133(27.5 \%)$ were anemic at the time of MDR-TB diagnosis.

Fifty-one $(10.1 \%)$ patients had chronic diseases other than HIV and anemia. Among other chronic diseases, 19 (3.7\%) had diabetes and 18 (3.7\%) had cardiac illness (Table 2).

3.3. Treatment Outcome. Of the 484 MDR-TB patients, 315 (65.1\%) were cured and $125(25.8 \%)$ completed treatment that makes the proportion of MDR-TB patients who successfully completed treatment 440 (90.1\%), 24 (5\%) dead, and $20(4.1 \%)$ lost follow-up (Table 3 ).

3.4. Survival Time. All 484 patients were followed up for a median of 247.5 days (IQR: 237 to 497.75 months) ranging from 2 days to 1340 days. The total person-days of follow-up was 158,337 days. During this period, 24 deaths were recorded, resulting in 1.5 deaths per 10,000 person-days. The median time to death was 57.5 days (IQR, 30-95 days), indicating $75 \%$ of deaths occurred during the first 95 days of the MDR-TB course of treatment.

The cumulative survival rate of MDR-TB patients at the end of treatment was $94.9 \%$. At six months of the MDT-TB course of treatment, the survival probability was $95.2 \%$. The cumulative failure probability of patients was high in the first six months of follow-up (Table 4 and Figures 1 and 2).

\subsection{Factors Associated with MDR-TB Death. As shown in} Table 5, with bivariable analysis, baseline weight, hemoglobin level, HIV status, previous history of treatment, clinical complication, and the presence of lung cavities were significantly associated with the time of death. However, in multivariable binary Cox regression, being anemic, HIV coinfection, and clinical complications were significantly associated with death during MDR-TB medication. Those who had clinical complications were more than 3 times at risk to have a death outcome than those who had no clinical complications $(\mathrm{AHR}=2.55$; 95\% CI: $1.06,6.10)$. HIV-infected patients were about 6-folds at risk to have a death outcome than noninfected MDR-TB patients $(\mathrm{AHR}=5.7$; 95\% CI: 2.2, 14.51), and anemic patients were more than 4folds at risk than nonanemic patients $(\mathrm{AHR}=4.26 ; 95 \% \mathrm{CI}$ : $1.65,11.02$ ) (Table 5).

\section{Discussion}

A retrospective cohort study was conducted among 484 MDR-TB patients in Saint Peter's Specialized Hospital, Addis Ababa, Ethiopia, to assess the survival status and predictors of mortality. A majority, 440 (90.9\%) (65.1\% cured and $25.8 \%$ completed), of the patients had successful treatment outcomes, which reflects Saint Peter's specialized Hospital has achieved the "Global Plan to END TB 2016-2020" target of $\geq 87 \%$ [18].

The survival rate of $94.85 \%$ of MDR-TB patients in St. Peter's Specialized Hospital has shown considerable improvement compared to the $78.95 \%$ report in the same hospital 7 years ago [6]. It is also higher than $88 \%$ in SNNPR [14] and $80 \%$ in Wuhan, China [16].The difference might be due to the change in time that is related to increased patients' awareness of their health and improvement in patient care.

The death rate was 1.5 per 10,000 person-days which is lower than the report of Wuhan, China, which was 1.79 per 10,000 person-days, SNNPR, Ethiopia, 1.92, and much lower than the incidence rate in this hospital before 7 years which was 4 per 10,000 person-days $[5,12,19]$. The possible reason for these findings is as follows: first, it might be because the setting is a TB-specialized hospital that has good infrastructure with highly trained and skilled healthcare workers and the site is a research center for tuberculosis that helps the hospital to identify gaps to intervene for good achievement. Second, chronic diseases get more emphasis that decreases the mortality of patients related to it.

Almost all deaths of patients, 23 (95.8\%), occurred in the first 6 months of the MDR-TB treatment course which is consistent with a study report in Northwest Ethiopia [11]. The following would be the possible explanations: Firstly, a 
TABLE 2: Clinical characteristics of MDR-TB patients in SPSH, $2020(n=484)$.

\begin{tabular}{|c|c|c|c|}
\hline Variables & & Frequency & Percent (\%) \\
\hline \multirow{4}{*}{ Methods of diagnosis } & Gene Xpert & 359 & 74.2 \\
\hline & Culture & 72 & 14.9 \\
\hline & Line probe assay & 46 & 9.5 \\
\hline & Clinically suspected & 7 & 1.4 \\
\hline \multirow{2}{*}{ Type of MDR-TB } & Pulmonary TB & 389 & 80.4 \\
\hline & Extra-pulmonary TB & 95 & 19.6 \\
\hline \multirow{2}{*}{ Initial sputum smear result $(n=389)$} & Positive & 87 & 22.4 \\
\hline & Negative & 302 & 77.6 \\
\hline \multirow{2}{*}{ Previous treatment for $\mathrm{TB}$} & Yes & 329 & 68.0 \\
\hline & No & 155 & 32.0 \\
\hline \multirow{3}{*}{ Baseline weight } & $<43 \mathrm{~kg}$ & 172 & 35.5 \\
\hline & $\geq 43 \mathrm{~kg}$ & 312 & 64.5 \\
\hline & Median (IQR) & $45.5(40-52)$ & - \\
\hline \multirow{5}{*}{ Substance use } & Nonusers & 363 & 75 \\
\hline & Alcohol & 105 & 21.7 \\
\hline & Khat & 14 & 2.9 \\
\hline & Khat and alcohol & 1 & 0.2 \\
\hline & Smoker & 1 & 0.2 \\
\hline \multirow{3}{*}{ Number of drug used for MDR-TB therapy } & 4 & 117 & 24.2 \\
\hline & 5 & 363 & 75.0 \\
\hline & $\geq 6$ & 4 & 0.8 \\
\hline \multirow{3}{*}{ Initiation of MDR-TB medication } & At the day of diagnosis & 392 & 81.0 \\
\hline & Within 10 days of diagnosis & 91 & 18.8 \\
\hline & After 30 days of diagnosis & 1 & 0.2 \\
\hline \multirow{2}{*}{ Drug adverse effects } & Yes & 463 & 95.7 \\
\hline & No & 21 & 4.3 \\
\hline \multirow{7}{*}{ Type of adverse effects $(n=463)$} & GI symptoms & 332 & 68.6 \\
\hline & Peripheral neuropathy & 139 & 28.7 \\
\hline & Hypothyroidism & 25 & 5.2 \\
\hline & Ototoxicity & 31 & 6.4 \\
\hline & Psychiatric symptoms & 33 & 6.8 \\
\hline & Hypokalemia & 118 & 24.4 \\
\hline & Drug-induced hepatitis & 44 & 9.1 \\
\hline \multirow{2}{*}{ Clinical complication } & Yes & 96 & 19.8 \\
\hline & No & 388 & 80.2 \\
\hline \multirow{5}{*}{ Type of complication } & Pneumonia & 59 & 61.5 \\
\hline & Hemoptysis & 16 & 16.7 \\
\hline & Pneumothorax & 13 & 13.5 \\
\hline & Arthritis & 7 & 7.3 \\
\hline & Cor pulmonale & 1 & 1.0 \\
\hline \multirow{2}{*}{ Presence of lung cavities $(n=389)$} & Yes & 57 & 14.7 \\
\hline & No & 332 & 85.3 \\
\hline
\end{tabular}

IQR: interquartile range.

diagnosis of MDR-TB and being hospitalized for 6 months might create psychosocial problems that led to a poor prognosis. Secondly, more than one-third were from out of Addis Ababa, so the distance might have led to feeling like a stranger and it would be difficult to trace defaulters. Lastly, the majority (66.7\%) of the patients who died were on ART, and taking many drugs might have affected their psychological wellness that led to poor prognosis.

Hemoglobin level was one of the factors associated with mortality of MDR-TB patients in this study. Anemic patients were more than 4-folds at risk of death during MDR-TB medication than nonanemic patients. This result is consistent with a study from Northwestern Ethiopia, Tanzania, and
Haiti that describes anemia was an associated factor to the death of patients $[11,20,21]$.

Another factor associated with death of patients was HIV/AIDS coinfection. HIV-infected patients were 5.7 times more at risk to die while on MDR-TB treatment. This is in line with findings in the Amhara Region of Ethiopia, SNNPR, Ethiopia, South Africa, and Latvia [5, 8, 12, 22, 23]. HIV/AIDS might compromise their immunity and lead to poor prognosis of their health status.

Those who had clinical complications were 2.55 times at risk of death than those who had no clinical complications. Other studies have also shown that MDR-TB patients with clinical complications were associated with an increased risk 
TABLE 3: Comorbidities among MDR-TB patients in SPSH, $2020(n=484)$.

\begin{tabular}{|c|c|c|c|}
\hline Variables & & Frequency & Percent (\%) \\
\hline \multirow{2}{*}{ HIV coinfection } & Yes & 96 & 19.8 \\
\hline & No & 388 & 80.2 \\
\hline \multirow{2}{*}{ ART regimen $(n=95)$} & TDF-3TC-EFV & 92 & 96.8 \\
\hline & AZT-3TC-EFV & 3 & 3.2 \\
\hline CD4 count $(n=96)$ & Median CD4 count in cells $/ \mathrm{mm}^{3}$ (IQR) & \multicolumn{2}{|c|}{$163.5(129.75,197.75)$} \\
\hline \multirow{3}{*}{ Anemia } & Yes & 133 & 27.5 \\
\hline & No & 351 & 72.5 \\
\hline & Median (IQR) & \multicolumn{2}{|c|}{$13.4(12.2,14.5)$} \\
\hline \multirow{2}{*}{ Comorbidities (other than HIV) } & Yes & 49 & 10.1 \\
\hline & No & 435 & 89.9 \\
\hline \multirow{5}{*}{ Types of comorbidities (other than HIV) $(n=49)$} & Diabetes & 19 & 38.8 \\
\hline & Cardiac disease & 18 & 36.8 \\
\hline & Hypertension & 8 & 16.3 \\
\hline & Asthma & 3 & 6.1 \\
\hline & Renal disease & 1 & 2.0 \\
\hline
\end{tabular}

TDF-3TC-EFV: tenofovir disoproxil fumarate-lamivudine-efavirenz, AZT-3TC-EFV: azidothymidine-lamivudine-efavirenz, IQR: interquartile range.

TABLe 4: Treatment outcome of MDR-TB patients in SPSH, 2020.

\begin{tabular}{|c|c|c|c|c|}
\hline Variables & Treatment outcome & Frequency & Percent & $95 \% \mathrm{CI}$ \\
\hline \multirow{5}{*}{ MDR-TB treatment outcome } & Cured & 315 & 65.1 & $61.0,69.4$ \\
\hline & Completed & 125 & 25.8 & $21.9,30.0$ \\
\hline & Successful treatment outcome & 440 & 90.9 & $88.2,93.4$ \\
\hline & Lost follow-up & 20 & 4.1 & $2.5,6.0$ \\
\hline & Death & 24 & 5.0 & $3.1,7.0$ \\
\hline \multirow{2}{*}{ Survival outcome } & Censored (0) & 460 & 95.0 & $93.0,96.9$ \\
\hline & Event (1) & 24 & 5.0 & $3.1,7.0$ \\
\hline
\end{tabular}

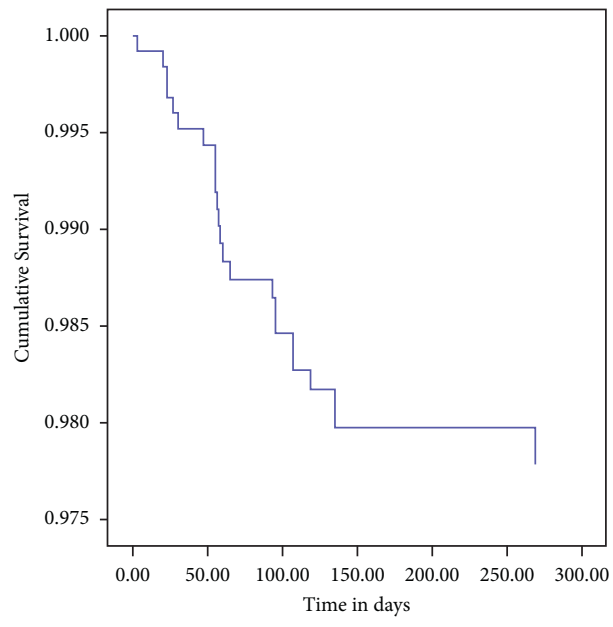

(a)

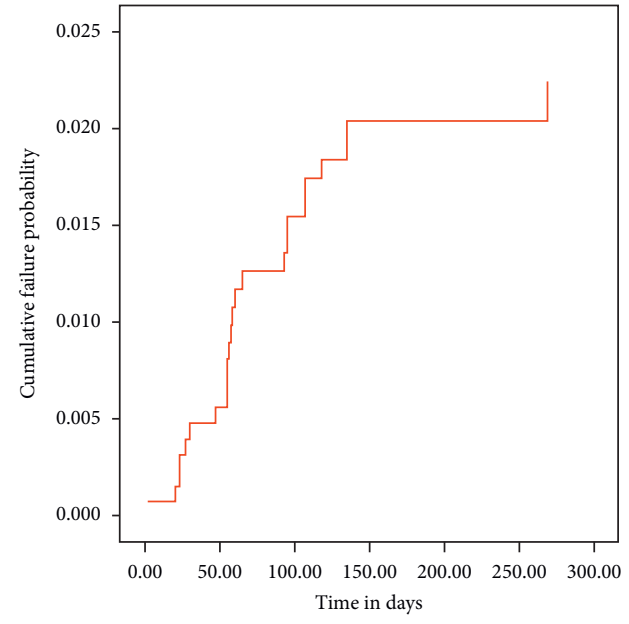

(b)

Figure 1: (a) Cumulative survival estimate and (b) cumulative failure probability of MDR-TB patients in St. Peter's Specialized Hospital, 2020.

of death $[5,19]$. Clinical complications may adversely affect the prognosis of patients involving the worsening of MDRTB and affect other organ systems.
This study had limitations of inability to control potentially important confounding variables such as drug adherence, economic status, nutritional status, and 


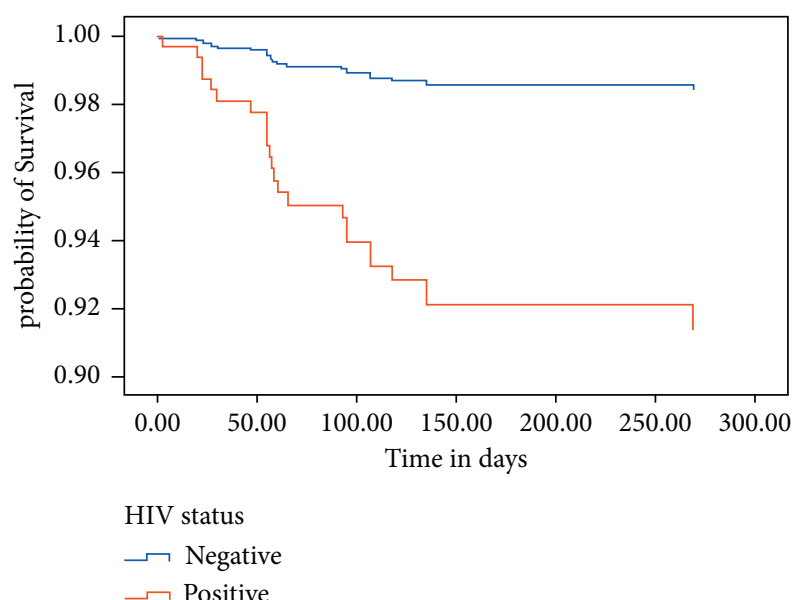

(a)

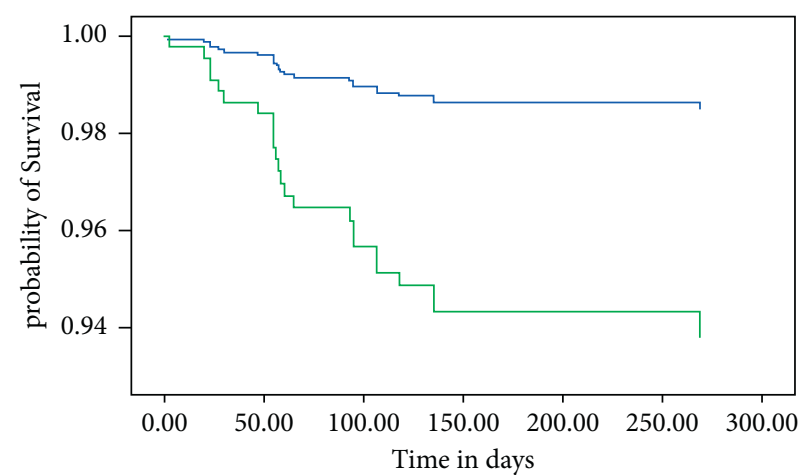

Anemia status

$\neg$ Normal

$\neg$ Anemic

(b)

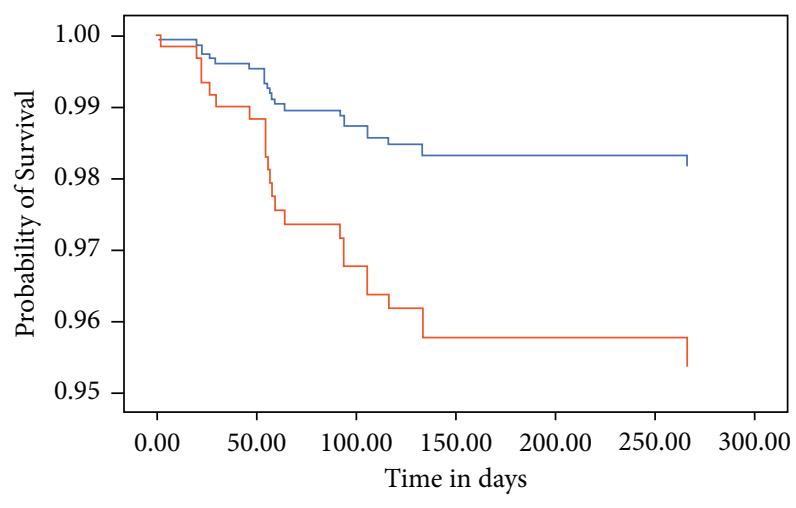

Clinical complication

$\neg$ No

$\neg$ Yes

(c)

Figure 2: Covariate adjusted survival function of MDR-TB patients in SPSH, 2020. (a) Stratified by HIV status, (b) stratified by anemia status, and (c) stratified by the presence of clinical complication.

TABLE 5: Cox proportional hazard regression analysis of predictors for time to death outcome among 484 MDR-TB patients in St. Peter's Specialized Hospital, 2020.

\begin{tabular}{|c|c|c|c|c|}
\hline Covariates & Event $^{\mathrm{d}}$ & Censored $^{\circ}$ & Crude HR & Adjusted HR \\
\hline \multicolumn{5}{|l|}{ Sex } \\
\hline Male & 8 & 228 & 1.00 & 1.00 \\
\hline Female & 16 & 232 & $1.94(0.83,4.53)^{-}$ & $1.56(0.62,3.93)$ \\
\hline \multicolumn{5}{|l|}{ Baseline weight } \\
\hline$<43 \mathrm{~kg}$ & 14 & 158 & $2.6(1.14,5.24)^{*}$ & $0.79(0.32,1.94)$ \\
\hline$\geq 43 \mathrm{~kg}$ & 10 & 302 & 1.00 & - \\
\hline \multicolumn{5}{|c|}{ Baseline hemoglobin } \\
\hline Anemic & 17 & 116 & $6.74(2.8,16.3)^{* * *}$ & $4.26(1.65,11.02)$ \\
\hline Not anemic & 7 & 344 & 1.00 & 1.00 \\
\hline \multicolumn{5}{|c|}{ Previous treatment for TB } \\
\hline Yes & 10 & 319 & 1.00 & 1.00 \\
\hline No & 14 & 141 & $3.1(1.37,6.95)^{* *}$ & $1.74(0.31,1.80)$ \\
\hline \multicolumn{5}{|c|}{ Clinical complication } \\
\hline Yes & 9 & 87 & $2.4(1.05,5.5)^{*}$ & $2.55(1.06,6.10)$ \\
\hline No & 15 & 373 & 1.00 & 1.00 \\
\hline HIV status & & & & \\
\hline
\end{tabular}


TABle 5: Continued.

\begin{tabular}{|c|c|c|c|c|}
\hline Covariates & Event $^{\mathrm{d}}$ & Censored $^{\circ}$ & Crude HR & Adjusted HR \\
\hline Positive & 16 & 80 & $8.65(3.7,20.2)^{* * *}$ & $5.7(2.2,14.51)$ \\
\hline Negative & 8 & 380 & 1.00 & 1.00 \\
\hline \multicolumn{5}{|c|}{ Presence of lung cavities } \\
\hline Yes & 5 & 52 & $2(0.75,5.4)^{\wedge}$ & $2.52(0.90,7.02)$ \\
\hline No & 19 & 408 & 1.00 & - \\
\hline
\end{tabular}

${ }_{\mathrm{d}}^{\mathrm{E}} \mathrm{E} e n t$ in this study was death; ${ }^{\circ}$ censored include cured, completed, and lost follow-up. ${ }^{* * *}=p<0.001,{ }^{* *}=p<0.01,{ }^{*}=p<0.05,{ }^{\wedge}=p<0.25$, HR $=$ hazard ratio.

educational factors which were not assessed to determine their relationship with the death of MDR-TB patients.

\section{Conclusions}

The probability of survival during MDR-TB medication was high that indicates St. Peter's Specialized Hospital has achieved 2020 milestones for a reduction in tuberculosisrelated deaths planed by the World Health Organization. Almost all deaths of MDR-TB patients were during the first six months of treatment. Being anemic, HIV coinfected cases, and clinical complications were associated with mortality from MDR-TB.

\section{Data Availability}

The data used to support the findings of this study are available from the corresponding author upon request.

\section{Ethical Approval}

Ethical clearance was obtained from the Institution Review Board of the Public Health Department, College of Health Science, Debre Berhan University. Then, a formal letter of cooperation was written to the Director of St. Peter's Specialized Hospital and permission was obtained. The information regarding any specific personal identifiers such as name of the patients was not collected, and also, the confidentiality of any personal information was maintained throughout the study process.

\section{Conflicts of Interest}

The authors declare no conflicts of interest.

\section{Authors' Contributions}

MA wrote the proposal, participated in data collection, analyzed the data, and drafted the manuscript. AB, BT, and AM approved the proposal with some revisions, participated in data analysis, and revised subsequent drafts of the paper. All authors read and approved the final manuscript.

\section{Acknowledgments}

The authors are very grateful to Debre Berhan University for approval of ethical clearance of this study. They would like to thank all data collectors in this study for their commitment in data extraction. Finally, they are also grateful to the Saint
Peter's Specialized Hospital staff, especially Abel Worku $(\mathrm{MPH})$, for their assistance and permission to undertake the research.

\section{References}

[1] World Health Organization, Global Tuberculosis Report 2019, World Health Organization, Geneva, Switzerland, 2019.

[2] ATS/CDC/ERS/IDSA, Prevention and Treatment of DrugResistant Tuberculosis: An Official Clinical Practice Guideline, 2019.

[3] World Health Organization, Multidrug-Resistant Tuberculosis (MDR-TB), 2017 Update, World Health Organization, Geneva, Switzerland, 2017.

[4] R. Reves and S. Angelo, As Ethiopia Moves toward Tuberculosis Elimination, Success Requires Higher Investment, Center for Strategic and International Studies, Washington, USA, 2016.

[5] T. Getachew, A. Bayray, and B. Weldearegay, "Survival and predictors of mortality among patients under multi-drug resistant tuberculosis treatment in Ethiopia: st. Peter's specialized tuberculosis hospital, Ethiopia," International Journal of Pharmaceutical Sciences and Research, vol. 4, 2013.

[6] Y. A. Limenih and D. L. Workie, "Survival analysis of time to cure on multi-drug resistance tuberculosis patients in Amhara region, Ethiopia," BMC Public Health, vol. 19, no. 1, p. 165, 2019.

[7] A. A. Woya, A. K. Tekile, and G. W. Basha, "Spatial frailty survival model for multidrug-resistant tuberculosis mortality in Amhara Region, Ethiopia," Tuberculosis Research and Treatment, vol. 8, 2019.

[8] T. Molie, Z. Teklemariam, E. Klinkenberg et al., "Intensive phase treatment outcome and associated factors among patients treated for multi drug resistant tuberculosis in Ethiopia: a retrospective cohort study," BMC Infectious Diseases, vol. 19, no. 1, p. 818, 2019.

[9] M. Verdecchia, K. Keus, S. Blankley et al., "Model of care and risk factors for poor outcomes in patients on multi-drug resistant tuberculosis treatment at two facilities in eSwatini (formerly Swaziland), 2011-2013," PLoS One, vol. 13, no. 10, 2018.

[10] Federal Democratic Republic of Ethiopia, Central Statistical Agency Population Projection of Ethiopia for All Regions at Wereda Level from 2014-2017, Federal Democratic Republic of Ethiopia, Addis Ababa, Ethiopia, 2013.

[11] K. A. Alene, K. Viney, E. S. McBryde, A. T. Tsegaye, and A. C. A. Clements, "Treatment outcomes in patients with multidrug-resistant tuberculosis in north-west Ethiopia," Tropical Medicine \& International Health, vol. 22, no. 3, pp. 351-362, 2017.

[12] T. Girum, Y. Tariku, and S. Dessu, "Survival status and treatment outcome of multidrug resistant tuberculosis (MDR- 
TB) among patients treated in treatment initiation centers (TIC) in South Ethiopia: a retrospective cohort study," Annals of Medical and Health Sciences Research, vol. 7, no. 5, 2017.

[13] D. Fantaw, M. Feyissa, S. Hamid, and W. Shibeshi, "Assessment of the survival status and risk factors for the mortality among multidrug resistant tuberculosis patients at Adama and Bishoftu general hospitals, Oromia, Ethiopia: a retrospective cohort study," Advances in Pharmacoepidemiology \& Drug Safety, vol. 7, no. 220, pp. 2167-1052, 2018.

[14] Federal Democratic Republic of Ethiopia MoH, "National guideline for TB, DR-TB and leprosy in Ethiopia," Federal Democratic Republic of Ethiopia $\mathrm{MoH}$, Addis Ababa, Ethiopia, 6 edition, 2018.

[15] D. G. Kleinbaum and M. Klein, Statistics for Biology and Health, M. Gail, K. Krickeberg, J. M. Samet, A. Tsiatis, and W. Wong, Eds., Springer Science+Business Media, LLC, New York, NY, USA, 3rd edition, 2012.

[16] Federal Democratic Republic of Ethiopia MoH, Guidelines for Clinical and Programmatic Management of Tb, Tb/hiv and leprosyin Ethiopia, Federal Democratic Republic of Ethiopia $\mathrm{MoH}$, Addis Ababa, Ethiopia, 5 edition, 2013.

[17] World Health Organization, Preventing and Controlling Iron Deficiency Anaemia through Primary Health Care: A Guide for Health Administrators and Programme Managers, World Health Organization, Geneva, Switzerland, 1989.

[18] World Health Organization, Global Plan to End TB 2016-2020-the Paradigm Shift, United Nations Office for Project Services, UNOPS, Geneva, Switzerland, 2016.

[19] J. Wang, M. Zhou, C. Chen et al., "Survival time and influencing factors in multidrug-resistant tuberculosis patients in Wuhan, 2006-2014," Zhonghua Liu Xing Bing Xue Za Zhi, vol. 40, no. 11, pp. 1409-1413, 2019.

[20] S. Isanaka, F. Mugusi, W. Urassa et al., "Iron deficiency and anemia predict mortality in patients with tuberculosis," Journal of Nutrition, vol. 142, no. 2, pp. 350-357, 2012.

[21] M. Charles, S. P. Koenig, S. C. Vilbrun et al., "Treatment outcomes for patients with multidrug-resistant tuberculosis in post-earthquake Port-au-Prince, Haiti," The American Journal of Tropical Medicine and Hygiene, vol. 91, no. 4, pp. 715-721, 2014.

[22] Y. Balabanova, O. Ignatyeva, L. Fiebig et al., "Survival of patients with multidrug-resistant TB in Eastern Europe: what makes a difference?” Thorax, vol. 71, no. 9, pp. 854-861, 2016.

[23] A. O. Olaleye and A. K. Beke, "Survival of smear-positive multidrug resistant tuberculosis patients in Witbank, South Africa: a retrospective cohort study," Infectious Diseases, vol. 48 , no. 6 , pp. 422-427, 2016. 